Pacific Journal of Mathematics

CONICALLY BOUNDED SETS IN BANACH SPACES 


\title{
CONICALLY BOUNDED SETS IN BANACH SPACES
}

\author{
RICHARD D. BOURGIN
}

\begin{abstract}
A condition on subsets of a Banach space $E$ is introduced, intermediate to those of norm and linear boundedness, which depends in an essential way on the topological as well as the linear structure of $E$. It is shown that this notion, called conical boundedness, is a strictly weaker notion than that of boundedness in some Banach spaces (including infinite dimensional reflexive spaces and infinite dimensional Banach spaces with separable duals) and coincides with that of boundedness in others (including $\ell_{1}$ and all finite dimensional spaces). After a discussion of some of the consequences of the condition of conical boundedness and a result on general structure of convex sets in reflexive spaces in terms of this notion, a construction is given which is valid in any nonreflexive Banach space and which yields two characterizations of reflexive Banach spaces. The first is in terms of (the nonexistence of) certain nonconically bounded convex sets, and the other descibes nonreflexive spaces via the restriction of any nonzero continuous linear functional to the unit balls of equivalent norms.
\end{abstract}

This latter result was first proved by Klee although his proof differs significantly from ours.

Since the notions of weak and norm boundedness in a Banach space coincide (and coincide with that of weak* boundedness if it is a dual Banach space) these seemingly disparate notions give, in fact, only one handle on the size of a set in such a space. Moreover since the condition of linear boundedness fails to take the topological structure into account, conical boundedness provides a proper topological linear space relaxation of boundedness.

I am very much indebted to Professor $\mathrm{Z}$. Zielezny for many stimulating conversations on the subject of this paper. (In particular a specific example of his contained the ideas involved in the proof of Proposition 13.) I also wish to thank Professor V. Drobot who was instrumental in the development of Proposition 7, and the referee for many helpful suggestions and especially for his "cleaning up" the construction.

We begin with a definition of conical boundedness.

1. Defininion. Let $B$ be a Banach space and $x \in B$. Let $\mathscr{C}(x)=$ $\{C \subset B: C$ is norm closed bounded and convex and $x \notin C\}$ and for each $C$ in $\mathscr{C}(x)$ denote by $K_{x}(C)$ the cone over $C$ with vertex $x$. (That is, $K_{x}(C)=\{t c+(1-t) x: t \geqq 0$ and $c \in C\}$.) $\quad$ A set $D \subset B$ is said to 
be conically bounded at $x$ if $D \cap K_{x}(C)$ is a bounded set for each $C \in \mathscr{C}(x)$. The set $D$ is conically bounded if there is a point $x \in B$ such that $D$ is conically bounded at $x$.

When $x=0$ we will henceforth write $K(C)$ in place of $K_{0}(C)$.

The first result concerns the role of the vertex $x$ of the cones in Definition 1.

2. Proposition. $A$ set $D$ is conically bounded at one point if and only if it is conically bounded at any other point. Hence $D$ is conically bounded if and only if it is conically bounded at 0.

Proof. By symmetry it suffices to show that if $D$ is conically bounded at 0 then it is conically bounded at $x \neq 0$. This will be accomplished by proving that whenever $C \in \mathscr{C}(x)$ there is a set $C_{1} \in$ $\mathscr{C}(0)$ for which $K_{x}(C) \backslash K\left(C_{1}\right)$ is bounded. Let $d_{1}=\inf \{\|c-x\|: c \in C\}$ and $t=3 d_{1}^{-1}\|x\|$. Then the set $C^{\prime}=\{t c+(1-t) x: c \in C\}$ is in $\mathscr{C}(x)$, $K_{x}\left(C^{\prime}\right)=K_{x}(C)$, and $\inf \left\{\left\|c^{\prime}\right\|: c^{\prime} \in C^{\prime}\right\} \geqq 2\|x\|$. Let $C_{1}$ be the closed convex hull of $C^{\prime} \cup\left(C^{\prime}-x\right)$. Note first that if $0 \leqq r \leqq 1$ and $c_{1}, c_{2} \in$ $C^{\prime}$ then

$$
\begin{aligned}
\left\|r\left(c_{1}-x\right)+(1-r) c_{2}\right\| & \geqq\left\|r c_{1}+(1-r) c_{2}\right\|-\|x\| \\
& \geqq 2\|x\|-\|x\|=\|x\|>0
\end{aligned}
$$

so that $0 \notin C_{1}$. Next let $d_{2}=\sup \left\{\left\|c^{\prime}-x\right\|: c^{\prime} \in C^{\prime}\right\}$. Then if $y \in K_{x}\left(C^{\prime}\right)$ and $\|y-x\|>d_{2}$, there is a number $s, 0<s<1$, such that $s y+(1-s) x \in$ $C^{\prime}$. Since

$$
s y=s(s y+(1-s) x)+(1-s)(s y+(1-s) x-x)
$$

the point $s y$ is a convex combination of a point of $C^{\prime}$ and one of $C^{\prime}-x$ so that $s y \in C_{1}$ and $y \in K\left(C_{1}\right)$. It follows that $K_{x}(C) \backslash K\left(C_{1}\right)=$ $K_{x}\left(C^{\prime}\right) \backslash K\left(C_{1}\right) \subseteq\left\{y:\|y-x\| \leqq d_{2}\right\}$ which completes the proof.

Later results provide large classes of examples of conically bounded sets (and of nonconically bounded linearly bounded convex sets) but the following elementary examples point out the strong dependence of this notion on the topological structure of the underlying Banach space.

3. ExAmple. Let $B$ be any of the Banach spaces $c_{0}$ or $l_{p}, 1 \leqq$ $p<\infty$. Let $e_{n}=(0,0, \cdots, 1,0, \cdots)$ be the point of $B$ with one in the $n$th place and 0 elsewhere and let $D=\left\{n e_{n}: n=1,2, \cdots\right\}$.

First of all suppose that $B$ is $c_{0}$ or $l_{p}, 1<p<\infty$. If $C \in \mathscr{C}(0)$ 
assume that $K(C) \cap D=\left\{n e_{n}: n \in A\right\}$ and that $A$ is infinite. For each $n \in A$ choose any positive number $d_{n}$ for which $d_{n} e_{n} \in C$ (so that $\left\{d_{n}: n \in A\right\}$ is bounded). Since $\left\{e_{n}\right\}_{n=1}^{\infty}$ converges in the weak topology on $B$ to 0 so does $\left\{d_{n} e_{n}\right\}_{n \in A}$ and hence $0 \in C$. From this contradiction we conclude that $K(C) \cap D$ is finite and hence $D$ is an unbounded, conically bounded set in $c_{0}$ and $l_{p}, 1<p<\infty$.

If $B=l_{1}$ then $D \subset K(C)$ where $C \in \mathscr{C}(0)$ is the set $C=\left\{\left(x_{i}\right) \in\right.$ $l_{1}: x_{i} \geqq 0$ for each $i$ and $\left.\sum_{j=1}^{\infty} x_{j}=1\right\}$. Hence $D$ is not conically bounded as a subset of $l_{1}$. (See Proposition 7 and the discussion following it.)

Among the operations which preserve bounded sets, many also preserve conically bounded ones although there are some important differences. (See Example 6). The next Proposition gives several such properties as well as an elementary but useful criterion on sets equivalent to that of conical boundedness. We will first need some notation.

4. Notation. Let $B$ be a Banach space, $\varepsilon>0$ and $f$ any nonzero element of $B^{*}$. Let

$$
C(f ; \varepsilon)=\{x \in B:\|x\| \leqq 1 \text { and } f(x) \geqq \varepsilon\}
$$

and

$$
K(f ; \varepsilon)=K(C(f ; \varepsilon)) .
$$

Furthermore, if $A \subset B$ let $\operatorname{conv} A$ [respectively, $\overline{\operatorname{conv}} A$ ] denote the convex hull [respectively, closed convex hull] of $A$, and $\overline{\mathrm{sp}} A$ the closed linear span of $A$.

5. Proposition. Let $B$ be a Banach space. The following statements are all concerned with subsets of $B$.

(a) Any subset of a conically bounded set is conically bounded.

(b) Finite unions of conically bounded sets are conically bounded.

(c) $D$ is conically bounded if and only if each of its countably infinite unbounded subsets is conically bounded.

(d) If $D$ is conically bounded, $t \in \boldsymbol{R}$, and $x_{0} \in B$, then $t D+x_{0}$ is conically bounded.

(e) If $D$ is conically bounded, $x_{0} \in B$, and $N$ any real number, then $\left\{t x+(1-t) x_{0}: t\right.$ is between 0 and $N$ and $\left.x \in D\right\}$ is conically bounded.

(f) If $D$ is conically bounded and $D_{1}$ is of finite distance from $D$ then $D_{1}$ is conically bounded. In particular the closure of a conically bounded set is conically bounded. $\left(D_{1}\right.$ is of finite distance from $D$ if there is a number $M$ for which inf $\{\|x-y\|: y \in D\} \leqq M$ for each 
$x$ in $\left.D_{1}\right)$.

(g) The closed convex hull of a bounded set and a conically bounded convex set is conically bounded.

(h) $D$ is conically bounded if and only if $D \cap K(f ; \varepsilon)$ is bounded for each $\varepsilon>0$ and nonzero $f$ in $B^{*}$.

Proof. Statements (a)-(e) follow directly from Definition 1 and, perhaps, an application of Proposition 2.

(f): For $C \in \mathscr{C}(0)$ define $C_{1}$ in $\mathscr{C}(0)$ by $C_{1}=\left\{3 M d^{-1} c: c \in C\right\}$ where $M$ is as in the statement of part (f) and $d=\inf \{\|c\|: c \in C\}>0$. Let $C_{2}=\left\{x \in B: \inf \left\{\|x-y\|: y \in C_{1}\right\} \leqq 2 M\right\}$ so that $C_{2} \in \mathscr{C}(0)$. If $x \in D_{1} \cap$ $K(C)$ either $\|x\| \leqq N_{1}=\sup \left\{\|c\|: c \in C_{1}\right\}$ or, if $\|x\|>N_{1}$ there is a number $t, 0<t<1$, such that $t x \in C_{1}$. But by hypothesis there is a point $y$ in $D$ with $\|x-y\|<2 M$ so that $\|t x-t y\|=t\|x-y\|<2 M$. That is, $t y \in C_{2}$ and hence $y \in K\left(C_{2}\right)$. Since $D \cap K\left(C_{2}\right)$ is bounded (say $\sup \left\{\|z\|: z \in D \cap K\left(C_{2}\right)\right\}=N_{2}$ ) we conclude that $\|x\| \leqq\|y\|+2 M \leqq$ $N_{2}+2 M$. Hence, in either case, if $x \in K(C) \cap D$ then $\|x\| \leqq \max \left(N_{1}\right.$, $N_{2}+2 M$ ) which completes the proof of this part.

(g): Let $A$ be a bounded set and $D$ a conically bounded convex set. For any point $x_{0}$ in $D$ let $d=\sup \left\{\left\|x_{0}-y\right\|: y \in A\right\}$. It is evident that the convex set $D_{1}=\{z: \inf \{\|z-x\|: x \in D\} \leqq d\}$ contains $D \cup A$. Since $D_{1}$ is closed and convex it contains $\overline{\text { conv }}(D \cup A)$. Finally, $D_{1}$ is conically bounded (by part (f)) and hence so is $\overline{\operatorname{conv}}(D \cup A$ ) (by part (a)).

(h): For any set $C$ in $\mathscr{C}(0)$ choose $f \in B^{*}$ and $\delta>0$ such that $0<\delta<\inf \{f(x): x \in C\}$. Let $M=\sup \{\|c\|: c \in C\}$ and let $\varepsilon=M^{-1} \delta$. If $x \in K(C)$ then $t x \in C$ for some $t>0$ and hence $\left\|M^{-1} t x\right\| \leqq 1$. But $f\left(M^{-1} t x\right)=M^{-1} f(t x) \geqq \varepsilon$ so $x \in K(f ; \varepsilon)$. Thus $K(C) \leqq K(f ; \varepsilon)=K(C(f ; \varepsilon))$ where $C(f ; \varepsilon)$ is obviously in $\mathscr{C}(0)$. This proves part (h).

6. ExAmple. The hypotheses of Proposition 5, part (g) are not superfluous as the following example (of a conically bounded set whose convex hull is not conically bounded) demonstrates.

Let $B$ be the Banach space $c_{0}$ and for $n=1,2 \cdots$ let $e_{n} \in c_{0}$ denote the $n^{\text {th }}$ unit vector (as in the previous Example 3). Then the set $D=\left\{n\left(e_{1} \pm n e_{n}\right) ; n=1,2 \cdots\right\}$ is conically bounded. In fact if $0 \neq$ $f=\left(f_{i}\right) \in \mathscr{L}_{1}=c_{0}^{*}$ and $\varepsilon>0$, clearly $\left\{n:(1+n)^{-1} f\left(e_{1} \pm n e_{n}\right) \geqq \varepsilon\right\}$ is finite since $\lim _{i \rightarrow \infty} f_{i}=0$. From $n\left(e_{1} \pm n e_{n}\right) /\left\|n\left(e_{1} \pm n e_{n}\right)\right\|=(1+n)^{-1}\left(e_{1} \pm n e_{n}\right)$ we conclude that there are only finitely many $n$ for which either $n\left(e_{1}+n e_{n}\right)$ or $n\left(e_{1}-n e_{n}\right)$ belongs to $K(f ; \varepsilon)$. It follows from Proposition 5 part (h) that $D$ is conically bounded. On the other hand $1 / 2\left[n\left(e_{1}+n e_{n}\right)\right]+1 / 2\left[n\left(e_{1}-n e_{n}\right)\right]=n e_{1} \in K\left(\left\{e_{1}\right\}\right)$ so that conv $(D)$ is not conically bounded.

We come now to a characterization of those Banach spaces in 
which each conically bounded set is bounded.

7. Proposition. In a Banach space $B$ the following conditions are equivalent:

(a) Each conically bounded subset of $B$ is bounded;

(b) Weak and norm convergences of sequence coincide.

Proof. not $(\mathrm{b}) \Rightarrow$ not (a): If there is a sequence $\left\{x_{n}\right\}$ which converges weakly to $x$ and yet for each $n$ we have $\left\|x_{n}-x\right\|>\delta$ for some $\delta>0$ then taking $y_{n}=\left\|x_{n}-x\right\|^{-1}\left(x_{n}-x\right)$, the sequence $\left\{y_{n}\right\}$ converges weakly to 0 and each $y_{n}$ has norm one. For any positive $\varepsilon$ and nonzero $f \in B^{*}$ certainly $n y_{n} \in K(f ; \varepsilon)$ implies that $y_{n} \in C(f ; \varepsilon)$ and this can happen for at most finitely many $n$ by choice of the sequence $\left\{y_{n}\right\}$. Thus $\left\{n y_{n}\right\} \cap K(f ; \varepsilon)$ is finite (hence bounded) and $\left\{n y_{n}\right\}$ is thus an unbounded, conically bounded set.

not $(a) \Rightarrow$ not $(b)$ : If there is a conically bounded but unbounded set in $B$, it contains a countable conically bounded subset $\left\{x_{n}\right\}_{n=1}^{\infty}$ with $\left\|x_{n}\right\| \geqq n$. for each $n$. Thus $\left\{x_{n}\right\} \cap K(f ; \varepsilon)$ must be finite for each $\varepsilon>0$ and $f \in B^{*}, f \neq 0$, so that $f\left(\left\|x_{n}\right\|^{-1} x_{n}\right)<\varepsilon$ for all but finitely many $n$. That is, for each $f \in B^{*}$ we have $\lim \sup f\left(\left\|x_{n}\right\|^{-1} x_{n}\right) \leqq 0$ and hence $\left\{\left\|x_{n}\right\|^{-1} x_{n}\right\}_{n=1}^{\infty}$ converges weakly to 0 . Since each point of this sequence has norm one, the proof is complete.

From Proposition 7 it follows that in finite dimensional spaces and in $l_{1}$ (see for example [2, Cor. 2, p. 33]) every conically bounded set is bounded, while every Banach space which has an infinite dimensional subspace with separable dual (and hence each infinite dimensional reflexive space) contains an unbounded, conically bounded subset.

The next result (and Corollary) provide a simple but descriptive restriction on the convex subsets of reflexive spaces.

8. Proposition. Let $E$ be a reflexive Banach space and $W \subset E$ a closed convex set which contains no (infinite) rays. Then $W$ is conically bounded.

Proof. Since $W$ is conically bounded if and only if $W^{n}-y$ is (for any point $y \in W$ ) we assume without loss of generality that $0 \in$ $W$. Then it suffices to show that $K(f ; \varepsilon) \cap W$ is bounded whenever $f \in B^{*}, f \neq 0$ and $\varepsilon>0$. If $\left\{x_{n}: n=1,2, \cdots\right\}$ lies in this intersection and $\left\|x_{n}\right\| \geqq n$ for each $n$ then $\left\{\left\|x_{n}\right\|^{-1} x_{n}: n=1,2, \cdots\right\}$ is a sequence in $C(f ; \varepsilon)$, a weakly compact set, so there is a point $x_{0}$ in $C(f ; \varepsilon)$ which is a cluster point of the sequence. (Of course $x_{0} \neq 0$ since $\left.f\left(x_{0}\right) \geqq \varepsilon_{0}\right)$ For any integer $N$ the point $N x_{0}$ is a cluster point of the 
sequence $\left\{N\left\|x_{n}\right\|^{-1} x_{n}: n=N, N+1, \cdots\right\}$ and since $W$ is convex, contains $x_{n}$ and 0 and $N\left\|x_{n}\right\|^{-1} \leqq 1$ for $n \geqq N$, the sequence $\left\{N\left\|x_{n}\right\|^{-1} x_{n}: n=\right.$ $N, N+1, \cdots\}$ lies in $W$. Since $W$ is (weakly) closed the point $N x_{0}$ must belong to $W$ for each positive integer $N$. That is, $W$ contains the ray $\left\{t x_{0}: t \geqq 0\right\}$, a contradiction which completes the proof.

9. Corollary. A convex set in a reflexive Banach space is conically bounded if an only if its closure contains no rays.

Proof. If $W$ is conically bounded then by Proposition 5, part (f) so is its closure, $\operatorname{cl}(W)$. But it is evident that no set containing a ray can be conically bounded, so in fact $\operatorname{cl}(W)$ contains no rays. The converse follows from Proposition 9, and from Proposition 5 part (a).

Proposition 8 shows that, in particular, if $W$ is any linearly bounded convex body which is symmetric about 0 in a reflexive Banach space $E$ then $W$ is conically bounded. (By 'convex body' we mean 'closed convex set with nonempty interior'.) Such sets are the unit balls of continuous norms on $E$, and the construction presented below shows that no such statement is possible for nonreflexive Banach spaces. In fact, we will prove somewhat more.

10. Proposition. Let $B$ be a nonreflexive Banach space and $0 \neq f \in B^{*}$. Then there is a continuous norm for $B$ whose unit ball $W$ satisfies: $\quad W \cap K(f ; \varepsilon)$ is unbounded for an appropriate $\varepsilon>0$. (Henee $W$ is not conically bounded.)

The proof of this proposition is a corollary of the construction. First, recall that a sequence $\left(x_{i}\right)_{i=1}^{\infty}$ is a basic sequence in a Banach space if to each point $x \in \overline{\mathrm{sp}}\left(x_{i}\right)_{i=1}^{\infty}$ there corresponds a unique sequence $\left(f_{i}(x)\right)_{i=1}^{\infty}$ of real numbers such that $x=\sum f_{i}(x) x_{i}$. It may be shown that the functionals $f_{i}$ so defined (called the associated biorthogonal functionals) are continuous linear functionals on $\overline{\mathrm{sp}}\left(x_{i}\right)_{i=1}^{\infty}$ and

$$
\sup \left\{\left\|x_{n}\right\|\left\|f_{n}\right\|: n=1,2, \cdots\right\}<\infty .
$$

(See [7, p. 1, p. 17, p. 23, and Th. 3.1, p. 20] for details.

11. The construction. Let $B$ be a nonreflexive Banach space and $0 \neq f \in B^{*}$. Note that $f^{-1}(0)$, being of codimension one in $B$, must itself be nonreflexive. It follows from a result of Pełczynski [5, Th. 2, p. 374] that there is a basic sequence $\left(y_{i}\right)_{i=1}^{\infty}$ with $0<$ $\inf \left\|y_{i}\right\| \leqq \sup \left\|y_{i}\right\| \leqq M<\infty$ for $\overline{\operatorname{sp}}\left(y_{i}\right)_{i=1}^{\infty}$ and $g_{1} \in\left[\overline{\operatorname{sp}}\left(y_{i}\right)\right]^{*}$ for which $\lim \sup \left|g_{1}\left(y_{i}\right)\right| \neq 0$. By passing to a subsequence if necessary and by 
possibly replacing $g_{1}$ by $-g_{1}$ one finds an $\varepsilon>0$ and a subsequence $\left(y_{i}^{\prime}\right)$ of $\left(y_{i}\right)$ for which $g_{1}\left(y_{i}^{\prime}\right) \geqq \varepsilon$ for $i=1,2, \cdots$. Let $x_{i}=\varepsilon\left(M g_{\mathrm{r}}\left(y_{i}^{\prime}\right)\right)^{-1} y_{i}^{\prime}$ and $g=M \varepsilon^{-1} g_{1}$. Then $\left(x_{i}\right)_{i=1}^{\infty}$ is a basis for $\overline{\operatorname{sp}}\left(x_{i}\right)_{i=1}^{\infty}$ with $\left\|x_{i}\right\| \leqq 1$ for each $i, g \in\left[\operatorname{sp}\left(x_{i}\right)_{i=1}^{\infty}\right]^{*}$, and $g\left(x_{i}\right)=1$ for $i=1,2, \cdots$.

Choose $x_{0} \in B$ such that $f\left(x_{0}\right)>0$ and note that $\left(x_{i}\right)_{i=0}^{\infty}$ is also a basic sequence. Let $\left.\left(f_{i}\right)\right)_{i=0}^{\infty}$ be the associated sequence of biorthogonal functionals (in $\left.\left[\overline{\mathrm{sp}}\left(x_{i}\right)_{i=0}^{\infty}\right]^{*}\right)$. Furthermore, let $\widetilde{f}_{i} \in B^{*}$ denote any HahnBanach extension of $f_{i}$ (so that $\left.\left\|f_{i}\right\|=\left\|\widetilde{f}_{i}\right\|\right)$ and choose an extension $\tilde{g} \in B^{*}$ of $g$ so that $\widetilde{g}\left(x_{0}\right)=-1$. Finally, denote by $q$ the natural quotient map $q: B \rightarrow B / \overline{\mathrm{sp}}\left(x_{i}\right)_{i=0}^{\infty}$.

It is evident that

$$
p(x)=|\widetilde{g}(x)|+\sup _{n=1,2, \cdots}\left|n^{-1} \tilde{f}_{n}(x)\right|+\|q(x)\|
$$

defines a seminorm on $B$. Since $\inf \left\{\left\|x_{i}\right\|: i=1,2, \cdots\right\}>0$ (since $\widetilde{g}\left(x_{i}\right)=1$ for each such $i)$ and $\sup \left\{\left\|x_{i}\right\|\left\|f_{i}\right\|: i=1,2, \cdots\right\}<\infty$, we conclude that $\lim _{n \rightarrow \infty}(1 / n)\left\|\widetilde{f}_{n}\right\|=0$. Consequently $p$ is continuous.

To see that $p$ is in fact a norm, suppose that $x \neq 0$. If $x \notin$ $\overline{\mathrm{sp}}\left(x_{i}\right)_{i=0}^{\infty}$ then $q(x) \neq 0$ and thus $p(x) \neq 0$. If $x \in \overline{\mathrm{sp}}\left(x_{i}\right)_{i=0}^{\infty}$ then $x=$ $\sum_{i=0}^{\infty} \tilde{f}_{i}(x) x_{i}$. If $\widetilde{f}_{n}(x) \neq 0$ for some $n \geqq 1$ then $\left|n^{-1} \widetilde{f}_{n}(x)\right| \neq 0$ and hence $p(x) \neq 0$. Otherwise $0 \neq x=\widetilde{f}_{0}(x) x_{0}$. Consequently $\widetilde{g}(x)=-\widetilde{f}_{0}(x) \neq$ 0 and again $p(x) \neq 0$. This completes the construction.

Proof of Proposition 10. With the notation developed in the Construction above, let $W=\{x: p(x) \leqq 1\}$. Then the set $S=\left\{n\left(x_{0}+x_{n}\right)\right.$ : $n=1,2, \cdots\}$ is unbounded and $S \subset W \cap K\left(f ; f\left(x_{0}\right) /\left(\left\|x_{0}\right\|+1\right)\right)$ since

$$
f\left(n\left(x_{0}+x_{n}\right)\right)=n f\left(x_{0}\right) \geqq \frac{f\left(x_{0}\right)}{\left\|x_{0}\right\|+1}\left\|n\left(x_{0}+x_{n}\right)\right\|
$$

for each $n \geqq 1$, thus completing the proof.

Propositions 8 and 10 combine to yield the following characterization of reflexive Banach spaces.

12. CoRollary. A Banach space is reflexive if and only if the unit ball of each continuous norm is conically bounded. That is, $B$ is reflexive if and only if each linearly bounded closed convex body symmetric about 0 is conically bounded.

Turning now to our second characterization of reflexive Banach spaces, recall that Bishop and Phelps [1] proved that for any Banach space $B$, the collection of elements of $B^{*}$ which attain their norm is a norm dense subset of $B^{*}$, while James [3] proved that if each $f \in$ $B^{*}$ attains its norm then $B$ is reflexive. A natural question related 
to these results is: What linear functionals on a Banach space $B$ attain their norms for each equivalent norm on $B$. If $B$ is reflexive then of course each $f \in B^{*}$ attains its norm for each equivalent norm. The complete answer for nonreflexive spaces is given in Proposition 13. We wish to thank Professor R. R. Phelps for informing us that Klee [4, Th. 1, p. 16] first proved this result (by different methods).

13. Proposition. $A$ Banach space $B$ is reflexive if and only if there is a nonzero element of $B^{*}$ which attains its norm for each equivalent norm on $B$.

Proof. Suppose that $(B, n)$ is not reflexive and $0 \neq f \in B^{*}$. Choose $x_{0} \in B$ for which $f\left(x_{0}\right)=1$ and let

$$
\|x\|=\max \left\{n\left(x-f(x) x_{0}\right),|f(x)|\right\} .
$$

It is easy to check that $\|\cdot\|$ is an equivalent norm on $B$ and that $\left\|x_{0}\right\|=\|f\|=f\left(x_{0}\right)=1$. Define $\left(x_{i}\right)_{i=1}^{\infty},\left(\widetilde{f}_{i}\right)_{i=0}^{\infty}, \tilde{g}$ and $p$ as in Construction 11 , and note that $p\left(x_{0}+x_{k}\right)=k^{-1}$ and $\left\|x_{0}+x_{k}\right\|=1$ (since $n\left(x_{k}\right) \leqq$ 1 for $k \geqq 1$ ).

Let $\|x\|=p(x)+\|x\|$. Then $\|\cdot\| \|$ is an equivalent norm for $B$ and we now show that $f$ does not attain its $\|\cdot \mid\|$ norm. If $\|x\| \mid \leqq 1$ then $\|x\|<1$ and hence $|f(x)|<1$. But for $k \geqq 1$ we have $1=f\left(x_{0}+x_{k}\right)$ and

$$
\left\|\left|x_{0}+x_{k}\left\|\mid=p\left(x_{0}+x_{k}\right)+\right\| x_{0}+x_{k} \|=k^{-1}+1 .\right.\right.
$$

It follows that $\|\mid f\|=1$, which completes the proof.

14. Remarks. (1). A notion bearing some resemblance to conical boundedness has been implicit in some work of Phelps [6]. His condition and ours are quite different, though, since he only requires boundedness in the direction of some $K(f ; \varepsilon)$ while we require boundedness in the direction of each $K(f ; \varepsilon)$.

(2). The definition of conical boundedness evidently carries over without change to general topological vector spaces, and thus a spectrum of problems are immediately raised.

\section{REFERENCES}

1. E. Bishop and R. R. Phelps. A proof that every Banach space is subreflexive, Bull. Amer. Math. Soc., 67 (1961), 97-98.

2. M. M. Day, Normed Linear Spaces, Ergebnisse der Mathematik und ihrer Grenzgebiete, Academic Press, New York, 1962.

3. R. C. James, Characterizations of reflexivity, Studia Math., 23 (1964), 205-216.

4. V. L. Klee, Some characterizations of reflexivity, Revista de Ciencias, 473-474 (1950), 15-23: 
5. A. Pelczynski, A note on the paper of I. Singer "Basic Sequences and reflexivity of Banach spaces", Studia Math., 21 (1962), 317-374.

6. R. R. Phelps, Support cones in Banach spaces and their applications, 1971 (to appear).

7. I. Singer, Bases in Banach Spaces, Die Grundlehren der mathematischen Wissenschaften, Band 154, Springer-Verlag, New York, 1970.

Received October 18, 1971 and in revised form March 15, 1972.

State University of New York at Buffalo 



\section{PACIFIC JOURNAL OF MATHEMATICS}

\section{EDITORS}

\section{H. SAMELSON}

Stanford University

Stanford, California 94305

C. R. Новву

University of Washington Seattle, Washington 98105

\section{J. DuGundJI}

Department of Mathematics University of Southern California Los Angeles, California 90007

RICHARD ARENS

University of California Los Angeles, California 90024

\section{ASSOCIATE EDITORS}
E. F. BECKENBACH
B. H. NeumanN
F. WOLF
K. YoSHIDA

\section{SUPPORTING INSTITUTIONS}

\author{
UNIVERSITY OF BRITISH COLUMBIA \\ CALIFORNIA INSTITUTE OF TECHNOLOGY \\ UNIVERSITY OF CALIFORNIA \\ MONTANA STATE UNIVERSITY \\ UNIVERSITY OF NEVADA \\ NEW MEXICO STATE UNIVERSITY \\ OREGON STATE UNIVERSITY \\ UNIVERSITY OF OREGON \\ OSAKA UNIVERSITY
}

\author{
UNIVERSITY OF SOUTHERN CALIFORNIA \\ STANFORD UNIVERSITY \\ UNIVERSITY OF TOKYO \\ UNIVERSITY OF UTAH \\ WASHINGTON STATE UNIVERSITY \\ UNIVERSITY OF WASHINGTON \\ $*{ }^{*}$
AMERICAN MATHEMATICAL SOCIETY
NAVAL WEAPONS CENTER
}

The Supporting Institutions listed above contribute to the cost of publication of this Journal, but they are not owners or publishers and have no responsibility for its content or policies.

Mathematical papers intended for publication in the Pacific Journal of Mathematics should be in typed form or offset-reproduced, (not dittoed), double spaced with large margins. Underline Greek letters in red, German in green, and script in blue. The first paragraph or two must be capable of being used separately as a synopsis of the entire paper. The editorial "we" must not be used in the synopsis, and items of the bibliography should not be cited there unless absolutely necessary, in which case they must be identified by author and Journal, rather than by item number. Manuscripts, in duplicate if possible, may be sent to any one of the four editors. Please classify according to the scheme of Math. Rev. Index to Vol, 39. All other communications to the editors should be addressed to the managing editor, Richard Arens, University of California, Los Angeles, California, 90024.

50 reprints are provided free for each article; additional copies may be obtained at cost in multiples of 50 .

The Pacific Journal of Mathematics is issued monthly as of January 1966. Regular subscription rate: $\$ 48.00$ a year (6 Vols., 12 issues). Special rate: $\$ 24.00$ a year to individual members of supporting institutions.

Subscriptions, orders for back numbers, and changes of address should be sent to Pacific Journal of Mathematics, 103 Highland Boulevard, Berkeley, California, 94708.

PUBLISHED BY PACIFIC JOURNAL OF MATHEMATICS, A NON-PROFIT CORPORATION

Printed at Kokusai Bunken Insatsusha (International Academic Printing Co., Ltd.), 270, 3-chome Totsuka-cho, Shinjuku-ku, Tokyo 160, Japan. 


\section{Pacific Journal of Mathematics}

\section{Vol. 44, No. $2 \quad$ June, 1973}

Tsuyoshi Andô, Closed range theorems for convex sets and linear liftings . . . . . . 393

Richard David Bourgin, Conically bounded sets in Banach spaces . . . . . . . . . 411

Robert Jay Buck, Hausdorff dimensions for compact sets in $R^{n} \ldots \ldots \ldots \ldots \ldots \ldots . \ldots 421$

Henry Cheng, A constructive Riemann mapping theorem ................ 435

David Fleming Dawson, Summability of subsequences and stretchings of

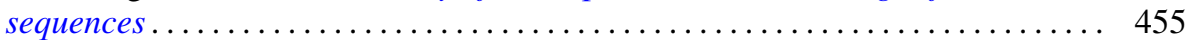

William Thomas Eaton, A two sided approximation theorem for 2-spheres ....... 461

Jay Paul Fillmore and John Herman Scheuneman, Fundamental groups of compact complete locally affine complex surfaces ....................... 487

Avner Friedman, Bounded entire solutions of elliptic equations . . . . . . . . . . . 497

Ronald Francis Gariepy, Multiplicity and the area of an $(n-1)$ continuous

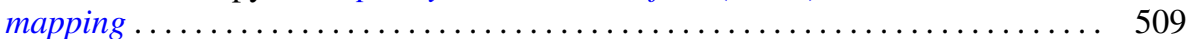

Andrew M. W. Glass, Archimedean extensions of directed interpolation groups . . . . 515

Morisuke Hasumi, Extreme points and unicity of extremum problems in $H^{1}$ on

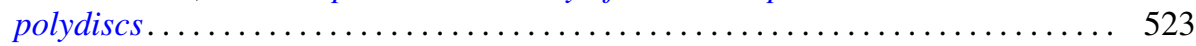

Trevor Ongley Hawkes, On the Fitting length of a soluble linear group . . . . . . 537

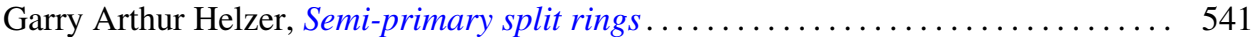

Melvin Hochster, Expanded radical ideals and semiregular ideals . . . . . . . . . 553

Keizō Kikuchi, Starlike and convex mappings in several complex variables . . . . . . 569

Charles Philip Lanski, On the relationship of a ring and the subring generated by its

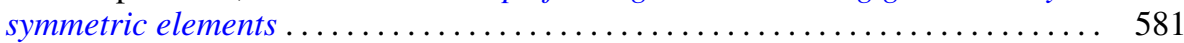

Jimmie Don Lawson, Intrinsic topologies in topological lattices and semilattices ........................................... 593

Roy Bruce Levow, Counterexamples to conjectures of Ryser and de Oliveira ...... 603

Arthur Larry Lieberman, Some representations of the automorphism group of an infinite continuous homogeneous measure algebra ..........

William George McArthur, $G_{\delta}$-diagonals and metrization theorems $\ldots .$.

James Murdoch McPherson, Wild arcs in three-space. II. An invariant of

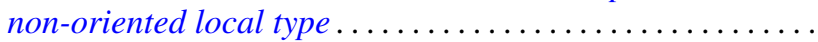

H. Millington and Maurice Sion, Inverse systems of group-valued measures ...

C. Edward Moore, Concrete semispaces and lexicographic separation of convex

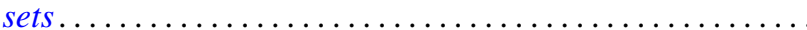

Jingyal Pak, Actions of torus $T^{n}$ on $(n+1)$-manifolds $M^{n+1}$.

Merrell Lee Patrick, Extensions of inequalities of the Laguerre and Turán type . . . . 675

Harold L. Peterson, Jr., Discontinuous characters and subgroups of finite index. . . . 683

S. P. Philipp, Abel summability of conjugate integrals . . . . . . . . . . . . . 693

R. B. Quintana and Charles R. B. Wright, On groups of exponent four satisfying an

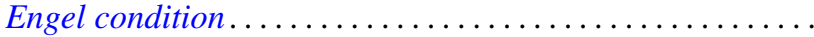

Marlon C. Rayburn, On Hausdorff compactifications. . . . . . . . . .

Martin G. Ribe, Necessary convexity conditions for the Hahn-Banach theorem in

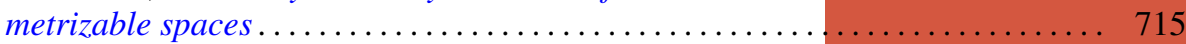

Ryōtarō Satō, On decomposition of transformations in infinite measure spaces .... 733

Peter Drummond Taylor, Subgradients of a convex function obtained from a

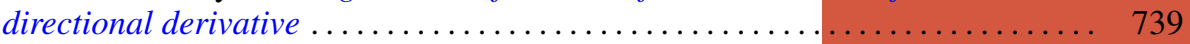

James William Thomas, A bifurcation theorem for $k$-set contractions . . . . . . . . 749 Clifford Edward Weil, A topological lemma and applications to real functions . . . . 757

Stephen Andrew Williams, A nonlinear elliptic boundary value problem . . ....... 767

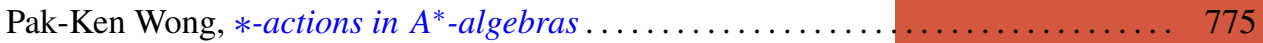

